\title{
Risiken und Ressourcen für die psychische Gesundheit von Kindern und Jugendlichen
}

\author{
U. Ravens-Sieberer ${ }^{1}$ \\ M. Schulte-Markwort ${ }^{2}$ \\ S. Bettge ${ }^{1}$ \\ C. Barkmann²
}

Risk and Protective Factors for Mental Health of Children and Adolescents

Zusammenfassung

Angesichts der vergleichsweise hohen Prävalenz psychischer Auffälligkeiten und Störungen bei Kindern und Jugendlichen weist die psychische Gesundheit als Zielgröße eine große Relevanz für die Public-Health-Forschung auf. Ziel der Zusatzstudie „Psychische Gesundheit“, die als Modul dem Kinder- und Jugendgesundheitssurvey des Robert Koch-Instituts angegliedert ist, ist es, die Prävalenz spezifischer psychischer Störungen bei Kindern und Jugendlichen in einer bundesweit repräsentativen Stichprobe differenziert zu ermitteln sowie Einflussgrößen auf die psychische Gesundheit im Entwicklungsverlauf zu untersuchen. In einer Unterstichprobe des Surveys, die ca. 4000 Kinder und Jugendliche im Alter von 7-17 Jahren umfasst, werden durch ein Screening psychische Auffälligkeiten erhoben. Eine einmalige Befragung erfasst die Häufigkeit des Auftretens von Risikofaktoren sowie vorhandene Ressourcen (protektive Faktoren) für psychische Gesundheit und liefert damit wertvolle Daten für die Gesundheitsberichterstattung (querschnittlicher Untersuchungsteil). Eine Subgruppe dieser querschnittlichen Stichprobe sowie eine zusätzliche klinische Stichprobe werden in einem Längsschnitt-Design wiederholt befragt, um die Effektstärken von Risiko- und protektiven Faktoren für psychische Gesundheit abzuschätzen und deren Zusammenwirken im Entwicklungsverlauf zu analysieren. Aus den Erkenntnissen werden Ansatzpunkte für wirksame Präventions- und Interventionsstrategien abgeleitet.

\section{Schlüsselwörter}

Epidemiologie · psychische Auffälligkeiten · Kinder und Jugendliche $\cdot$ Risikofaktoren $\cdot$ Schutzfaktoren

\begin{abstract}
Children's and adolescents' mental health is an epidemiological outcome of considerable public health relevance. This is based on a comparably high prevalence of mental health problems and disorders in children and adolescents. The supplementary study 'Mental Health', which is a modular part of the Health Survey for Children and Adolescents conducted by the Robert Koch Institute, aims at assessing the prevalence of specific mental health problems and disorders in a representative sample of children and adolescents in Germany. Furthermore, determinants of mental health will be studied in a developmental context. Screening to assess mental health problems will be performed in a representative sub-sample of the National Health Survey for Children and Adolescents. The sub-sample will comprise approximately 4,000 children and adolescents aged 7 to 17 years. Participants are interviewed once to assess the occurrence of risk factors and available assets (protective factors) for mental health, thus providing valuable data for health monitoring and planning (cross-sectional part of the study). A sub-group of this cross-sectional sample as well as an additional clinical sample are repeatedly studied in a longitudinal design. This part of the study is carried out to estimate the effect size of risk and protective factors on mental health, and to analyse their combined effects on the developmental process. From the findings, clues to effective prevention and intervention strategies can be derived.
\end{abstract}

\section{Key words}

Epidemiology $\cdot$ mental health problems $\cdot$ children and adolescents $\cdot$ risk factors $\cdot$ protective factors 


\section{Psychische Störungen und ihre Einflussfaktoren im Kindes- und Jugendalter}

Psychische Auffälligkeiten oder Störungen bei Kindern und Jugendlichen wie Aufmerksamkeitsdefizit-/Hyperaktivitätsstörungen, Depression oder Störungen des Sozialverhaltens werden in den Medien breit diskutiert. Eine Analyse vorhandener Daten zum Gesundheitszustand von Kindern durch die Bundeszentrale für gesundheitliche Aufklärung [1998] ergab, dass Entwicklungsund Gesundheitsrisiken bei Kindern zunehmen und dass psychosozial bedingte Befindlichkeitsstörungen ansteigen.

Internationale Studien zu Schätzungen der Gesamtprävalenz von psychischen Auffälligkeiten und Störungen im Kindes- und Jugendalter belegen, dass bei methodisch sorgfältig geplanten Untersuchungen mit klinisch erfahrenen Beurteilern international vergleichbare Ergebnisse berichtet werden. Der Mittelwert der beschriebenen Periodenprävalenzen in einem Überblick zur Epidemiologie psychischer Störungen im Kindes- und Jugendalter von Ihle und Esser [2002] beträgt 18\%. Auch in Deutschland liegt die Prävalenz psychischer Auffälligkeiten im Kindes- und Jugendalter mit ca. $18 \%$ seit Jahren stabil auf einem recht hohen $\mathrm{Ni}-$ veau [Döpfner et al. 1997, Barkmann, Schulte-Markwort 2002]. Damit stellt die psychische Gesundheit von Kindern und Jugendlichen eine epidemiologische Zielgröße erheblicher Relevanz dar. Allerdings weisen die Prävalenzraten internationaler und nationaler epidemiologischer Studien aufgrund vielfältiger methodischer Probleme erhebliche Schwankungen auf. Fragen der repräsentativen Stichprobenziehung, der Entwicklung valider und reliabler Erhebungsinstrumente sowie die Definition bzw. Operationalisierung und Klassifizierung psychischer Störungen des Kindes- und Jugendalters beeinträchtigen die Vergleichbarkeit. Neuere epidemiologische Studien bestätigen die schon früher immer wieder entdeckten deutlichen Beurteilereffekte [Döpfner et al. 1997, Barkmann, Schulte-Markwort 2002]. So schätzen Eltern ihre Kinder im Altersbereich zwischen 11 und 18 Jahren signifikant weniger belastet und auffällig ein als die Kinder und Jugendlichen sich persönlich im Selbsturteil.

Im Gegensatz zu den Prävalenzraten ist die tatsächliche Inanspruchnahme sehr viel geringer, weshalb sich aus häufig verwendeten Inanspruchnahmestatistiken kaum Rückschlüsse auf die tatsächliche Prävalenz psychischer Störungen ziehen lassen. Nur ca. $15-20 \%$ der Eltern von auffälligen Kindern und Jugendlichen nehmen eine Beratung oder Behandlung in Anspruch [Lehmkuhl et al. 1998], wodurch sich eine gravierende Unterversorgung psychischer Störungen abzeichnet. Psychische Erkrankungen sind oft langwierig, verursachen hohe Kosten im Gesundheitswesen und gehen mit deutlichen Beeinträchtigungen für die Betroffenen einher. Dies hat in Deutschland bisher noch nicht die nötige Aufmerksamkeit gefunden, so dass die Erfassung von psychischer Gesundheit im Kindes- und Jugendalter in epidemiologischen Studien und bevölkerungsrepräsentativen Untersuchungen im Rahmen von Public Health bisher ungerechtfertigterweise vernachlässigt wurde. Dies ist vor allen Dingen angesichts dessen, dass die meisten psychischen Störungen des Erwachsenenalters ihren Ursprung in Störungen im Kindes- und Jugendalter haben, zu bedauern. Dem Lebensabschnitt Kindheit und Jugend sollte daher eine größere Bedeutung für Prävention und Intervention und somit auch für die Einsparung von Folgekosten zukommen.
Gerade im Hinblick auf die Ausarbeitung gezielter Präventionsund Interventionsmaßnahmen ist es von besonderer Bedeutung, nicht nur das Vorhandensein psychischer Störungen zu betrachten, sondern vor allem die Wirkungsweise potenzieller Risikofaktoren und Ressourcen für die psychische Gesundheit in die Planung solcher Programme mit einzubeziehen. Als Risikofaktoren für psychische Erkrankungen werden biologische und psychosoziale Belastungen angeführt [Egle et al. 2002]. Auswirkungen derartiger Risiken auf die Gesundheit von Kindern und Jugendlichen wurden in Entwicklungsstudien nachgewiesen (Mannheimer Risikokinderstudie [Laucht et al. 1998]; Bayerische Entwicklungsstudie [Wolke, Meyer 1999]; Rostocker Längsschnittstudie [Meyer-Probst, Reis 1999]; Bielefelder und Regensburger Längsschnittstudie [Zimmermann et al. 1999]).

In den Gesundheitswissenschaften geraten neben der Betrachtung von Risikofaktoren für physische und psychische Erkrankungen zunehmend stärker auch Schutzfaktoren (Ressourcen) ins Blickfeld. Dabei setzt sich immer mehr die Sichtweise durch, dass neben pathogenen Bedingungen auch salutogene relevant sind für körperliche und seelische Gesundheit bzw. Störungen [Rutter 2000]. Den theoretischen Rahmen, in dem die Wirkungsweisen von sozialen Risiken und protektiven Faktoren auf die psychische Gesundheit von Kindern und Jugendlichen untersucht werden, bildet die Resilienzforschung [Masten et al. 1995]. Resilienz beschreibt „die Fähigkeit einer Person ..., relativ unbeschadet mit den Folgen beispielsweise belastender Lebensumstände umgehen und Bewältigungskompetenzen entwickeln zu können“ [Antonovsky 1991]. Im Entwicklungsverlauf entsteht Resilienz aus dem Zusammenwirken individueller und sozialer Eigenschaften und Lebensumstände von Kindern und Jugendlichen, die sich positiv auf Gesundheit und Wohlbefinden auswirken können. Diese werden als Ressourcen bezeichnet. In der empirischen Literatur zu Ressourcen für die psychische Gesundheit von Kindern und Jugendlichen wird eine Reihe von Faktoren genannt, für die sich protektive Wirkungen belegen lassen. Dabei können personale, familiäre und soziale Ressourcen unterschieden werden [Scheithauer, Petermann 1999].

Belastungen wie auch Ressourcen dürfen nicht isoliert betrachtet werden, sondern sie sind untereinander korreliert und wirken jeweils kumulativ [van Aken et al. 1996]. Zudem gibt es Wechselwirkungen von Risikofaktoren und gestörter Entwicklung [Meyer-Probst, Reis 1999] wie auch von Ressourcen und positiven Entwicklungsoutcomes [Masten, Reed 2001]. Hinsichtlich des Zusammenwirkens von Belastungen und Ressourcen wird in der Resilienzforschung angenommen, dass Ressourcen zur Bewältigung von Belastungen eingesetzt werden und somit die Wirkung von Risikofaktoren moderieren und abpuffern [Masten, Reed 2001]. Demnach erweisen sich Ressourcen in ihrer protektiven Wirkung erst angesichts von Risiken und widrigen Lebensumständen und bewirken so Resilienz. Zu Effektstärken von Belastungen und Ressourcen liegen erst vereinzelt empirische Ergebnisse vor. Während Beutel [1989] in seinem Überblick verschiedener Studien von konsistenten mittleren Effekten berichtet, fanden Laucht et al. [1998] nur kleine Risikofaktoren-Effekte und sehr kleine Ressourcen-Effekte. 
Obwohl Studien zur Prävalenz von psychischen Störungen vorliegen und die Auswirkungen vorliegender Risikofaktoren auf die Gesundheit von Kindern und Jugendlichen nachgewiesen wurden, existieren keine großen epidemiologischen Studien, die eine Verknüpfung der Prävalenz psychischer Auffälligkeiten im Kindes- und Jugendalter mit biologischen, familiären und sozialen Risiken einerseits und personalen, familiären und sozialen Ressourcen andererseits im Zeitverlauf erlauben. Diese Verknüpfung erfolgt in einem geplanten Modul als Anbindung an den ersten deutschen Kinder- und Jugendgesundheitssurvey [Kurth et al. 2002] und wird bedeutsame Aufschlüsse über die Entstehung und den Verlauf von psychischer Gesundheit bzw. psychischen Störungen von Kindern und Jugendlichen erbringen.

\section{Ziele und Fragestellung}

Ziel des Moduls „Risiken und Ressourcen für die psychische Gesundheit von Kindern und Jugendlichen“ ist es, Erkenntnisse zur Prävalenz psychischer Störungen im Kindes- und Jugendalter sowie zu Prävalenz, Wirkungsweisen und Effektstärken risikoerhöhender und protektiver Faktoren hinsichtlich Entstehung und Aufrechterhaltung psychischer Auffälligkeiten zu gewinnen. Hierzu sollen die im Rahmen des Kinder- und Jugendgesundheitssurveys erhobenen Eckwerte zu psychischen Auffälligkeiten, Belastungen und Ressourcen ergänzt und an einer Untergruppe der UntersuchungsteilnehmerInnen sowie an einer klinischen Stichprobe von Kindern und Jugendlichen mit bereits diagnostizierten psychischen Störungen vertiefend untersucht werden. Das Modul hat sich die Beantwortung folgender Hauptfragen zum Ziel gesetzt:

Wie groß ist die Prävalenz spezifischer psychischer Störungen im Kindes- und Jugendalter? Der Kernsurvey liefert allgemeine Screeningdaten zum Vorliegen psychischer Auffälligkeiten der untersuchten Kinder und Jugendlichen. Im Modul sollen diese Daten durch weitere Datenerhebungen vertieft und ergänzt werden hinsichtlich der Prävalenzbestimmung spezifischer psychischer Störungen. Der Schwerpunkt liegt hier besonders auf der Erfassung von Daten zum Vorliegen depressiver Störungen und zu Aufmerksamkeitsdefizit-/Hyperaktivitätsstörungen.

Welche Risiken und Ressourcen lassen sich bei Kindern und Jugendlichen bezogen auf die psychische Entwicklung identifizieren? Im Zentrum der Zusatzstudie steht die Frage nach den Auswirkungen von Belastungen, denen Kinder und Jugendliche ausgesetzt sind, auf ihre psychische Gesundheit sowie nach Ressourcen, über die sie verfügen. Es wird geprüft werden, in welcher Häufigkeit Kombinationen der untersuchten Belastungen und Ressourcen bei den Kindern und Jugendlichen auftreten. Zusätzlich werden Wechselwirkungen zwischen Belastungen und Ressourcen analysiert.

Wie wirken Belastungen und Ressourcen im zeitlichen Verlauf? Bei der Beantwortung dieser Frage geht es darum, wie sich Belastungen und Ressourcen im Entwicklungsverlauf auswirken und wie sie zur Entstehung oder Aufrechterhaltung psychischer Störungen beitragen. Hierbei werden Kinder und Jugendliche mit behandelten und unbehandelten Verläufen verglichen. Aus den Erkenntnissen über Wechselwirkungen und Effektstärken können Ansatzpunkte für gezielte Präventions- und Interventionsmaßnahmen abgeleitet werden.

\section{Design und Methodik}

Im Kinder- und Jugendgesundheitssurvey mit der repräsentativen Stichprobe von ca. 18000 Untersuchungsteilnehmern im Alter von 0 bis 17 Jahren können für das Gesamtspektrum gesundheitsrelevanter Fragestellungen bei Kindern und Jugendlichen lediglich Eckwerte erfasst werden [Kurth et al. 2002]. Daher wurde für den Kinder- und Jugendgesundheitssurvey von vornherein eine modulare Erweiterung eingeplant, um über ausgelagerte Zusatzbefragungen oder -untersuchungen Möglichkeiten zur vertiefenden Informationsgewinnung zu nutzen. Durch eine gemeinsame Nutzung der so gewonnenen konjunkten Daten von Kernsurvey und Modul entsteht die Möglichkeit, das Informationspotenzial des Gesamtsurveys zu erhöhen. Die „Modulpartner“ bemühen sich jeweils um eigenständige Finanzierung der Zusatzuntersuchungen.

Das Modul „Psychische Gesundheit“ nutzt die im Kernteil des Kinder- und Jugendgesundheitssurveys erhobenen Eckwerte zu psychischen Auffälligkeiten und subjektivem Wohlbefinden und ergänzt diese um differenzierte und spezifische Fragestellungen und zusätzliche Instrumente. Von Anfang an wurde darauf geachtet, dass eine enge Verzahnung des Moduls mit dem Kernsurvey gewährleistet ist, um eine Belastung der UntersuchungsteilnehmerInnen möglichst gering zu halten. Für das Modul wird aus allen Probanden im Altersbereich von 7 bis 17 Jahren, die am Kernsurvey teilnehmen, eine Unterstichprobe zufällig ausgewählt und bei ihrem Survey-Untersuchungstermin über diesen zusätzlichen Untersuchungsteil informiert. Jüngere Kinder von 0 bis 6 Jahren werden ausgeschlossen, da bei Einbeziehung dieses Altersspektrums keine einheitlichen und vergleichbaren Datenerhebungsverfahren und Untersuchungsinstrumente vorliegen. Die Modul-TeilnehmerInnen werden ergänzend zu der einmaligen Survey-Untersuchung zunächst querschnittlich von geschulten InterviewerInnen befragt; sie werden dafür zu von ihnen selbst angegebenen Terminen für die Befragung telefonisch kontaktiert. Von allen Teilnehmern am querschnittlichen Untersuchungsteil des Vorhabens wird zusätzlich das Einverständnis zur Teilnahme am längsschnittlichen Untersuchungsteil eingeholt. In diesem werden Wirkungsweisen und Effektstärken von Risiko- und Schutzfaktoren hinsichtlich des Auftretens bzw. der Aufrechterhaltung psychischer Störungen untersucht. Dazu werden die TeilnehmerInnen nach einem Jahr und nach zwei Jahren mit den gleichen Methoden wiederholt befragt. Die mehrfache Befragung in zeitlichen Abständen von einem Jahr im längsschnittlichen Untersuchungsteil erlaubt Aussagen über differenzielle Verlaufskurven der gesundheitlichen Entwicklung der Kinder und Jugendlichen.

Ergänzt wird die Survey-Unterstichprobe durch eine klinische Stichprobe, die ebenfalls in den längsschnittlichen Untersuchungsteil einbezogen und prospektiv verfolgt wird. In die klinische Stichprobe werden konsekutiv in kooperierenden kinderund jugendpsychiatrischen Kliniken teilstationär oder stationär neu aufgenommene Kinder und Jugendliche im Alter von 7 bis 17 Jahren einbezogen. Während des Klinikaufenthalts wird durch ein persönliches Interview eine klinische Diagnose gestellt. Die im Survey zu erfassenden somatischen Variablen und die Variablen aus dem Survey-Fragebogen werden ebenfalls während des klinischen Aufenthalts registriert. Nach der Entlassung beginnt die 
längsschnittliche Befragung parallel zur Survey-Unterstichprobe. Aus der klinischen Stichprobe gehen alle TeilnehmerInnen - ihr Einverständnis vorausgesetzt - in den längsschnittlichen Untersuchungsteil ein und werden ebenfalls im Abstand von jeweils einem Jahr mit den gleichen Verfahren telefonisch befragt. Die Einbeziehung der Inanspruchnahmen-Stichprobe lässt erwarten, dass mehr Probanden mit hohen Risiken und einer eher geringen Ausprägung von Ressourcen erreicht werden, so dass für diese Untergruppe genauere Abschätzungen der Wirkungsweisen adversiver und protektiver Faktoren möglich werden. Abb. 1 zeigt den Ablauf des Moduls im Überblick.

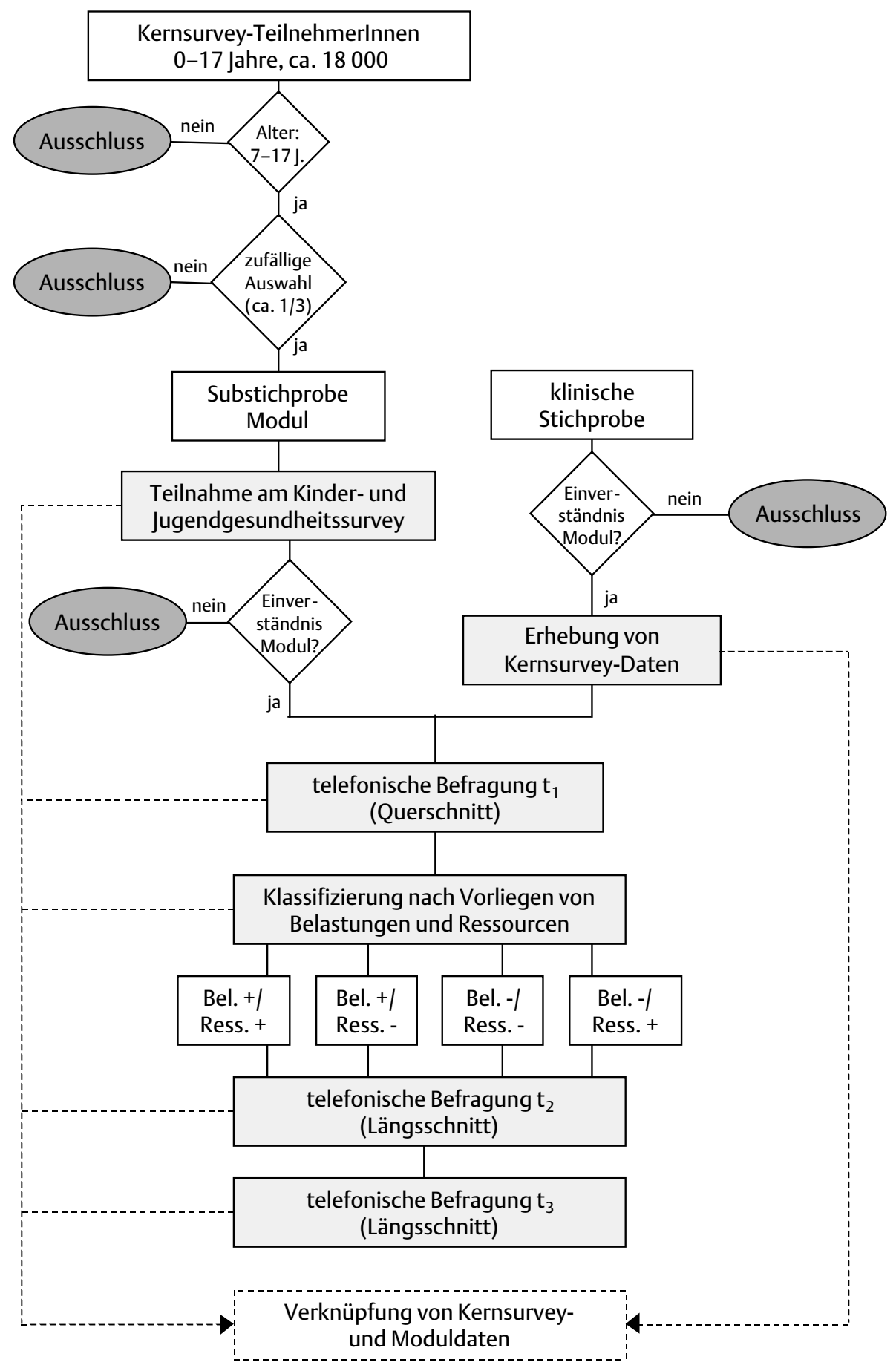

Die Methodenkombination von schriftlicher und telefonischer standardisierter Befragung hat sich im Pretest des Kinder- und Jugendgesundheitssurveys als praktikabel und hinsichtlich der Teilnahmebereitschaft als äußerst günstig erwiesen. Mit der zur Verfügung stehenden Technik für computerassistierte Telefoninterviews (CATI) sind telefonische Befragungen zeitsparend und kostengünstig zu realisieren. Die Daten werden während des Interviews am Computer erfasst und stehen damit unmittelbar nach Abschluss der Befragung für Analysen zur Verfügung. Telefoninterviews für Gesundheitssurveys werden zunehmend eingesetzt [Centers for Disease Control and Prevention 1997] und liefern mit schriftlichen Befragungsmethoden vergleichbare gute Ergebnisse [Fischer et al. 2001]. Als Informationsquelle wird sowohl das Fremdurteil eines Elternteils ( 7 bis 17 Jahre) als auch

Abb. 1 Design und Untersuchungsablauf. 
das Selbsturteil des Kindes bzw. Jugendlichen (11 bis 17 Jahre) genutzt. Das kindliche Selbsturteil wird durch die Perspektive der Eltern ergänzt, relativiert und kreuzvalidiert.

Die Merkmalsbereiche, die in enger Verzahnung von Kernsurvey und Modul erfasst werden, sind folgende:

1. psychische Auffälligkeiten (i.S. von Erlebens- und Verhaltensauffälligkeiten)

2. Belastungen/Risikofaktoren (biologische, familiäre, psychosoziale Merkmale und im Längsschnitt zwischenzeitliche Ereignisse)

3. Ressourcen/Schutzfaktoren (personale, familiäre und soziale Ressourcen)

\section{Erfassung psychischer Auffälligkeiten}

Im Fragebogen des Kinder- und Jugendgesundheitssurveys wird zur Gewinnung von Eckdaten zu psychischen Auffälligkeiten der „Strengths and Difficulties Questionnaire“ (SDQ) [Goodman 1997] eingesetzt. Dieses Instrument umfasst 25 Fragen auf fünf Skalen und liegt in einer Elternform für die Altersgruppe 4 bis 16 Jahre und einer Selbstberichtsform für Jugendliche (Altersgruppe 11 bis 16 Jahre) vor. Vorliegende Ergebnisse mit dem SDQ weisen auf seine Eignung als Screening-Instrument zur Erfassung psychischer Auffälligkeiten hin [Klasen et al. 2000]. In dem Modul wird zur vertiefenden Erfassung spezifischer psychischer Auffälligkeiten und Störungen die deutsche Version der Child Behavior Checklist ( $\mathrm{CBCL}$ ) als mehrdimensionales Instrument eingesetzt [Arbeitsgruppe Deutsche Child Behavior Checklist 1998a, Arbeitsgruppe Deutsche Child Behavior Checklist 1998b]. Sie erfasst im Selbstbericht (Youth Self Report - YSR, 11-18 Jahre) und im Elternbericht (6-18 Jahre) psychische Auffälligkeiten und Störungen auf acht Syndrom-Skalen mit insgesamt 112 Items, die zu externalisierenden, internalisierenden und sonstigen Störungen zusammengefasst werden können. Für den gesamten Altersbereich existieren deutsche Normwerte aus einer 1996 national repräsentativ durchgeführten Befragung. Zur Erfassung des Schwere- und Ausprägungsgrades einer depressiven Störung wird das Depressions-Inventar für Kinder und Jugendliche (DIKJ) [Stiensmeier-Pelster et al. 1989] eingesetzt. Das Verfahren erfasst im Selbsturteil mittels 27 Items depressive Symptome und ist für 8- bis 17-Jährige geeignet. Es existieren Normwerte für die deutsche Population. Außerdem wird das Vorliegen einer hyperkinetischen Störung geprüft. Dies geschieht mittels der Conners' Scale [Conners 1996], die in einer aus 10 Items bestehenden Kurzform eingesetzt wird und geschlechtsdifferenzierte Normwerte aus einer amerikanischen Referenzstichprobe aufweist. Die Conners' Scale basiert auf den im amerikanischen Sprachraum häufiger eingesetzten Kategorien des Diagnosesystems DSM-IV, weshalb die für die Diagnosestellung nach dem in Europa gewöhnlich verwendeten Klassifikationssystem ICD-10-relevanten Merkmale anhand der Beurteilungsbogen zur Hyperaktivität aus dem diagnostischen System DISYPS-KJ [Döpfner, Lehmkuhl 2000] ergänzt werden.

\section{Erfassung von Belastungen und Risikofaktoren}

Die Risikofaktoren lassen sich in biologische, familiäre und psychosoziale Einflussgrößen unterteilen. Als biologische Risikofaktoren können die Frühgeburtlichkeit sowie andere prä- und perinatale Risiken über die im Kinder- und Jugendgesundheitssurvey enthaltenen Fragen zu Schwangerschaft und Geburt erfasst wer- den. Eine belastende Familiensituation als familiäres Risiko wird mithilfe des Family Adversity Index (FAI) [Rutter, Quinton 1977] erhoben. Zur Erfassung elterlicher Belastungen wird die aus der Symptom-Checkliste (SCL-90-R) entwickelte Kurzform mit neun Items verwendet [Brähler, Klaghofer 2001]. Die elterlich wahrgenommene eigene Gesundheit erfasst die 12-Item-Kurzform des Medical Outcome Study SF-36 Health Surveys [Bullinger, Kirchberger 1998]. Aus den sich gegenseitig ergänzenden Informationen des Kernsurveys und der Modulbefragung wird ein kumulativer familiärer Risikoindex gebildet. Hinsichtlich der psychosozialen Risikofaktoren kann ein niedriger sozioökonomischer Status durch den aus Routinefragen des Kinder- und Jugendgesundheitssurveys gebildeten Schichtindex nach Winkler identifiziert werden. Für die Beurteilung der sozialen Lage aus Sicht der Jugendlichen selbst steht mit der Family Affluence Scale [Currie 2000] ein kurzes, international bewährtes Instrument zur Verfügung. Die genannten psychosozialen Risikofaktoren werden um weitere Items aus multiaxialen Diagnosesystemen der Kinder- und Jugendpsychiatrie ergänzt [Remschmidt, Schmidt 1994].

\section{Erfassung von Ressourcen und Schutzfaktoren}

Ergänzend zu den Eckwerten zu Ressourcen im Kernteil des Surveys (vor allem Selbstwirksamkeit, soziale Unterstützung und Familienklima) werden im Modul Instrumente zur Erfassung personaler, familiärer und sozialer Ressourcen vertiefend eingesetzt. Zur Erhebung personaler Ressourcen dienen Skalen zum Selbstwert und zum Optimismus, zusätzlich findet die Children's Sense of Coherence Scale (CSOC) [Kern et al. 1995] in Anlehnung an das salutogenetische Konzept von Antonovsky [1991] Anwendung. Im Bereich familiärer Ressourcen wird der elterliche Erziehungsstil im Elternurteil durch die Hamburger Erziehungsverhaltensliste für Mütter (HAMEL) erfasst, ein mit 24 Items ökonomisches Verfahren, welches die beiden Hauptdimensionen Unterstützung und Strenge erhebt. Das Instrument ist psychometrisch geprüft und es liegen Normwerte vor [Heller, Perleth 1991]. Im Selbsturteil der Jugendlichen werden die Survey-Fragen im Modul um Skalen zum elterlichen Monitoring, zum Bestrafungsverhalten, zur elterlichen Unterstützung und zum familiären Zusammenhalt [WHO 2001] ergänzt. Diese Operationalisierung ermöglicht die Erfassung der wesentlichen Bestandteile des als protektiv geltenden autoritativen Erziehungsstils, nämlich Involvement und Monitoring, sowie zusätzlich mit der Skala Bestrafungsverhalten die Abweichung von diesem als ideal konzipierten Erziehungsstil. Durch Items aus der Social Attributes Checklist [McClellan, Katz 2001] zur sozialen Kompetenz und zu Beziehungen zu Gleichaltrigen werden im Elternurteil Informationen über die von den familiären Ressourcen abzugrenzenden sozialen Ressourcen erhoben. Für die Erfassung des Selbsturteils der Jugendlichen zu sozialen Ressourcen werden im Modul ergänzend kurze Skalen zur peerbezogenen sozialen Kompetenz, zu Freundschaften und zum Schulklima aus der internationalen Studie „Health Behaviour in School aged Children“ (HBSC) [WHO 2001] verwendet. 


\section{Erwartete Ergebnisse}

Mit den für den Kernteil geplanten und im Pretest erprobten Methoden des Kinder- und Jugendgesundheitssurveys sollte es möglich sein, Eckwerte und Anhaltspunkte für die Prävalenz psychischer Auffälligkeiten im Kindes- und Jugendalter und für Teilbereiche personaler, familiärer und sozialer Ressourcen von Kindern und Jugendlichen zu erfassen und mithilfe sozioökonomischer Variablen Familien in problematischen Lebenslagen zu identifizieren. Das Modul „Risiken und Ressourcen für die psychische Gesundheit von Kindern und Jugendlichen" ermöglicht eine Erweiterung und Vertiefung dieser Aussagen zur psychischen Gesundheit aus dem Kernteil des Kinder- und Jugendgesundheitssurveys. Im geplanten querschnittlichen Modulteil werden zusätzliche bundesweite Daten zur psychischen und subjektiven Gesundheit, vor allem zur Prävalenz der besonders häufigen Störungsformen Depression und Aufmerksamkeitsdefizit-/Hyperaktivitätsstörung, sowie zur Ausprägung von Determinanten psychischer Gesundheit (Risikofaktoren, personale, soziale und familiäre Ressourcen) in der Altersgruppe der Schulkinder und Jugendlichen ermittelt. Dies erlaubt eine Identifikation von Risikogruppen mit Interventionsbedarf. Die Möglichkeit der Verknüpfung mit im Kernsurvey erhobenen somatischen Daten eröffnet eine weitergehende, ganzheitliche Perspektive auf das bio-psycho-soziale Gesundheitsgeschehen im Kindes- und Jugendalter. Somatische und psychische Komorbiditäten können übergreifend erfasst und in den Analysen berücksichtigt werden.

Der längsschnittliche Untersuchungsteil ermöglicht die Analyse von Unterschieden zwischen nicht mit Risiken belasteten Kindern und Jugendlichen, einer bevölkerungsbezogenen und einer klinischen Risikogruppe im zeitlichen Verlauf der psychischen und subjektiven Gesundheit. Innerhalb dieses Längsschnittdesigns werden die (belastenden und protektiven) Wirkweisen und Effektstärken von Determinanten psychischer Gesundheit im Zeitverlauf erfasst. Die längsschnittliche Untersuchung erlaubt die Prüfung aus der Resilienz-Forschung abgeleiteter prädiktiver Modelle zur Wirkung von Belastungen und Ressourcen im Kontext familiärer und sozialer Settings und Umstände auf die Entwicklung psychischer Gesundheit und psychischer Auffälligkeiten. Die Einbeziehung einer aus der Survey-Population gewonnenen Risikogruppe (hohe Belastungen, niedrige Ressourcen) und der klinischen Stichprobe psychisch auffälliger Kinder und Jugendlicher erlaubt differenzierte Aussagen über Wirkungsweisen und die Stärke des Einflusses belastender und protektiver Faktoren im Vergleich des „naturalistischen“ mit dem behandelten Verlauf.

Der Beitrag einzelner Belastungen und Ressourcen sowie ihr komplexes Zusammenwirken bei der Entwicklung psychischer Auffälligkeiten wurde bisher in epidemiologischen Studien nicht hinreichend berücksichtigt und bedeutet eine Ergänzung zu bestehenden repräsentativen Studien zur Erfassung der Prävalenz psychischer Störungen im Kindes- und Jugendalter [Döpfner et al. 1997, Wittchen et al. 1998]. Aus den Ergebnissen sowohl des querschnittlichen als auch des längsschnittlichen Designs unter Berücksichtigung der Effekte der beschriebenen und spezifizierten Risikofaktoren und Schutzfaktoren für psychische Gesundheit lässt sich eine detaillierte Beschreibung einer Risikopopulation vornehmen. Damit wird deutlich, dass eine Zusatzuntersuchung zur psychischen Gesundheit von Kindern und Jugendlichen in
Deutschland zum bundesweiten Kinder- und Jugendgesundheitssurvey eine bedeutsame klinische, deskriptiv epidemiologische und gesundheitspolitische Aufwertung des Gesamtvorhabens bedeutet. Die geplante enge Verknüpfung der Variablen des Kernsurveys mit dem Modul bietet nicht nur die Möglichkeit, an einer repräsentativen Stichprobe das somatische Störungs- und Erkrankungsspektrum zeitgleich mit einer differenzierten Erfassung psychischer Störungen und vorhandener Belastungen und Ressourcen zu verbinden, sondern ermöglicht darüber hinaus die Betrachtung der Wirkungsweisen von Determinanten der psychischen Gesundheit im Längsschnitt über den Zeitverlauf. Auf diese Weise können bedeutsame Aufschlüsse über die Entstehung und den Verlauf der psychischen Gesundheit bzw. psychischer Auffälligkeiten und Erkrankungen von Kindern und Jugendlichen gewonnen werden.

Weiterhin besteht die Möglichkeit, die in dem Modul erhobenen Daten zur psychischen Gesundheit, zur gesundheitsbezogenen Lebensqualität und zu personalen und sozialen Ressourcen sowie Belastungen von Kindern und Jugendlichen mit anderen Forschungsansätzen zu ähnlichen Fragestellungen in Beziehung zu setzen, da die gewählten Instrumente auf eine nationale und internationale Vergleichbarkeit abgestimmt sind. Die dargestellten Forschungsfragen, die Auswahl der Methoden und die erwarteten Daten stellen eine Ergänzung und Erweiterung bereits existierender epidemiologischer Studien im Kinder- und Jugendbereich dar.

\section{Literatur}

${ }^{1}$ Antonovsky A. Meine Odyssee als Stressforscher. Jahrbuch für Kritische Medizin 17. Berlin: Argument 1991; 112-130

${ }^{2}$ Arbeitsgruppe Deutsche Child Behavior Checklist. Elternfragebogen über das Verhalten von Kindern und Jugendlichen; deutsche Bearbeitung der Child Behavior Checklist (CBCL/4-18). Einführung und Anleitung zur Handauswertung. 2. Auflage mit deutschen Normen, bearbeitet von Döpfner M, Plück J, Bölte S et al. Köln: Arbeitsgruppe Kinder-, Jugend- und Familiendiagnostik (KJFD) 1998a

${ }^{3}$ Arbeitsgruppe Deutsche Child Behavior Checklist. Fragebogen für Jugendliche; deutsche Bearbeitung der Youth Self-Report Form der Child Behavior Checklist (YSR). Einführung und Anleitung zur Handauswertung. 2. Auflage mit deutschen Normen, bearbeitet von Döpfner M, Plück J, Bölte S et al. Köln: Arbeitsgruppe Kinder-, Jugend- und Familiendiagnostik (KJFD) 1998b

${ }^{4}$ Barkmann C, Schulte-Markwort M. Wie gesund sind die Seelen unserer Kinder? Zur Epidemiologie von Erlebens- und Verhaltensauffälligkeiten bei 4-18jährigen in Deutschland. In: Lehmkuhl U (Hrsg). Seelische Krankheit im Kindes- und Jugendalter - Wege zur Heilung. Abstractband XXVII. Berlin: Kongress der Deutschen Gesellschaft für Kinder- und Jugendpsychiatrie und Psychotherapie 3.-6. April, 2002

5 Beutel M. Was schützt Gesundheit? Zum Forschungsstand und der Bedeutung von personalen Ressourcen in der Bewältigung von Alltagsbelastungen und Lebensereignissen. Psychother Psych Med 1989; 39: 452-462

${ }^{6}$ Brähler E, Klaghofer R. Konstruktion und teststatistische Prüfung einer Kurzform der SCL-90-R. Zeitschrift für klinische Psychologie, Psychiatrie und Psychotherapie 2001; 49: 115-124

7 Bullinger M, Kirchberger I. SF-36. Fragebogen zum Gesundheitszustand. Z Klin Psychol-Forse 1998; 28: 143-145

8 Bundeszentrale für gesundheitliche Aufklärung (Hrsg). Gesundheit von Kindern. Epidemiologische Grundlagen. Köln: Forschung und Praxis der Gesundheitsförderung 1998; Band 3

${ }^{9}$ Centers for Disease Control and Prevention (CDC). Health Risks in America: Gaining Insight from the Behavioral Risk Factor Surveillance System. Atlanta, GA: U.S. Department of Health and Human Services, Public Health Service, Centers for Disease Control and Prevention, 1997. 
${ }^{10}$ Conners K. Conners' Rating Scale - Revised Technical Manual. New York: Multi-Health Systems Inc 1996

${ }^{11}$ Currie C. Health and well-being and their association with reported socio-economic circumstances among school-aged children in Europe and North America: the WHO HBSC study. In: Vleminckx K, Smeeding $\mathrm{T}$ (Hrsg). Child Well-being, Child Poverty and Child Policy in Modern Nations. UK: Policy Press 2000

${ }^{12}$ Döpfner M, Plück J, Berner W et al. Psychische Auffälligkeiten von Kindern und Jugendlichen in Deutschland - Ergebnisse einer repräsentativen Studie: Methodik, Alters-, Geschlechts- und Beurteilereffekte. Z Kinder Jug-Psych 1997; 25: 218-233

${ }^{13}$ Döpfner M, Lehmkuhl G. Diagnostik-System für psychische Störungen im Kindes- und Jugendalter nach ICD-10 und DSM-IV (DISYPS-KJ). 2. erweiterte Auflage. Bern: Huber 2000

${ }^{14}$ Egle UT, Hardt J, Franz M et al. Psychosoziale Belastungen in der Kindheit und Gesundheit im Erwachsenenalter. Psychotherapeut 2002; 47: $124-127$

${ }^{15}$ Fischer R, Meyer N, Weitkunat R et al. Population-based health monitoring via computer-assisted telephone interviews in Bavaria. Gesundheitswesen 2001; 63: 123-129

${ }^{16}$ Goodman R. The Strengths and Difficulties Questionnaire: A research note. J Child Psychol Psyc 1997; 38: 581-586

${ }_{17}$ Heller PA, Perleth C. Die Hamburger Erziehungsverhaltensliste für Mütter (HAMEL) von F. Baumgärtel. In: Keller KA (Hrsg). Begabungsdiagnostik in der Schul- und Erziehungsberatung. Bern: Huber 1991

${ }^{18}$ Ihle W, Esser G. Epidemiologie psychischer Störungen im Kindes- und Jugendalter: Prävalenz, Verlauf, Komorbidität und Geschlechtsunterschiede. Psychol Rundsch 2002; 53: 159-169

${ }^{19}$ Kern R, Rasky E, Noack RH. Indikatoren für Gesundheitsförderung in der Volksschule. Forschungsbericht 95/1 aus dem Institut für Sozialmedizin. Graz: Karl-Franzens-Universität 1995

${ }^{20}$ Klasen H, Woerner W, Wolke D et al. Comparing the German versions of the Strengths and Difficulties Questionnaire (SDQ-Deu) and the Child Behavior Checklist. Eur Child Adoles Psy 2000; 9: 271-276

${ }^{21}$ Kurth BM, Bergmann KE, Dippelhofer A et al. Die Gesundheit von Kindern und Jugendlichen in Deutschland. Was wir wissen, was wir nicht wissen, was wir wissen werden. Bundesgesundheitsbl - Gesundheitsforsch - Gesundheitsschutz 2001; 45: 852-858

${ }^{22}$ Laucht M, Esser G, Schmidt MH. Risiko- und Schutzfaktoren der frühkindlichen Entwicklung: Empirische Befunde. Z Kinder Jug-Psych 1998; 26: 6-20

${ }^{23}$ Lehmkuhl G, Döpfner M, Plück J et al. Häufigkeit psychischer Auffälligkeiten und somatische Beschwerden bei vier- bis zehnjährigen Kindern in Deutschland im Urteil der Eltern - ein Vergleich normorientierter und kriterienorientierter Modelle. Z Kinder Jug-Psych 1998; 26: 83-96
${ }^{24}$ Masten AS, Coatsworth JD, Neemann J et al. The structure and coherence of competence from childhood through adolescence. Child Dev 1995; 66: 1635-1659

${ }^{25}$ Masten AS, Reed MG. Resilience in Development. In: Snyder CR, Lopez SJ (Hrsg). The Handbook of Positive Psychology. Oxford: University Press 2001

${ }^{26}$ McClellan DE, Katz LG. Assessing young children's social competence. ERIC Digest, EDO-PS-01-2. University of Illinois, Clearinghouse on Elementary and Early Childhood Education 2001

${ }^{27}$ Meyer-Probst B, Reis O. Von der Geburt bis 25: Rostocker Längsschnittstudie (ROLS). Kindheit und Entwicklung 1999; 8: 59-68

${ }^{28}$ Remschmidt H, Schmidt MH. Multiaxiales Klassifikationsschema für psychische Störungen des Kindes- und Jugendalters nach ICD-10 der WHO. Bern: Huber 1994

${ }^{29}$ Rutter M, Quinton D. Psychiatric disorder - ecological factors and concepts of causation. In: McGurk M (Hrsg). Ecological factors in human development. Amsterdam: North-Holland 1977; 173-187

${ }^{30}$ Rutter M. Psychosocial influences: Critiques, findings and research needs. Dev Psychopathol 2000; 12: 375-405

${ }^{31}$ Scheithauer H, Petermann F. Zur Wirkungsweise von Risiko- und Schutzfaktoren in der Entwicklung von Kindern und Jugendlichen. Kind Entwickl 1999; 8: 3-14

32 Stiensmeier-Pelster J, Schürmann M, Duda K. Depressionsinventar für Kinder und Jugendliche; DIKJ. Göttingen: Hogrefe 1989

${ }^{33}$ van Aken MAG, Asendorpf JB, Wilpers S. Das soziale Unterstützungsnetzwerk von Kindern: Strukturelle Merkmale, Grad der Unterstützung, Konflikt und Beziehungen zum Selbstwertgefühl. Psychol Erz Unterr 1996; 43: 114-126

${ }^{34}$ WHO (Hrsg). Research Protocol for the HBSC 2001/02 Survey. Unveröffentl. Manuskript, 2001

${ }^{35}$ Wittchen HU, Nelson CB, Lachner G. Prevalence of mental disorders and psychosocial impairments in adolescents and young adults. Psychol Med 1998; 28: 109-126

${ }^{36}$ Wolke D, Meyer R. Ergebnisse der Bayerischen Entwicklungsstudie: Implikationen für Theorie und Praxis. Kindh Entwickl 1999; 8: 23-35

37 Zimmermann P, Suess GJ, Scheuerer-Englisch $\mathrm{H}$ et al. Bindung und Anpassung von der frühen Kindheit bis zum Jugendalter: Ergebnisse der Bielefelder und Regensburger Längsschnittstudie. Kindh Entwickl 1999; 8: $36-48$ 